\title{
The development of prospective memory in young schoolchildren: The impact of ongoing task absorption, cue salience, and cue centrality
}

\author{
Matthias Kliegel $^{\mathrm{a}, *}$, Caitlin E.V. Mahy ${ }^{\mathrm{a}, \mathrm{e}}$, Babett Voigt ${ }^{\mathrm{a}}$, Julie D. Henry ${ }^{\mathrm{b}}$, \\ Peter G. Rendell ${ }^{\mathrm{c}}$, Ingo Aberle ${ }^{\mathrm{d}}$ \\ ${ }^{a}$ Department of Psychology, University of Geneva, CH-1211 Geneva 4, Switzerland \\ ${ }^{\mathrm{b}}$ School of Psychology, University of Queensland, Brisbane, QLD 4072, Australia

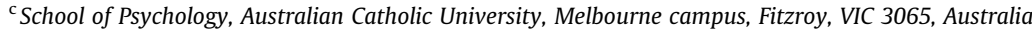 \\ ${ }^{\mathrm{d}}$ Department of Psychology, Technische Universität Dresden, D-01069 Dresden, Germany \\ ${ }^{\mathrm{e}}$ Department of Psychology, University of Oregon, Eugene, OR 97403, USA
}

\section{A R T I C L E I N F O}

\section{Article history:}

Received 16 October 2012

Revised 24 July 2013

\section{Keywords:}

Prospective memory

Ongoing task

Cue salience

Cue centrality

Schoolchildren

Development

\begin{abstract}
A B S T R A C T
This study presents evidence that 9- and 10-year-old children outperform 6- and 7-year-old children on a measure of event-based prospective memory and that retrieval-based factors systematically influence performance and age differences. All experiments revealed significant age effects in prospective memory even after controlling for ongoing task performance. In addition, the provision of a less absorbing ongoing task (Experiment 1), higher cue salience (Experiment 2), and cues appearing in the center of attention (Experiment 3) were each associated with better performance. Of particular developmental importance was an age by cue centrality (in or outside of the center of attention) interaction that emerged in Experiment 3. Thus, age effects were restricted to prospective memory cues appearing outside of the center of attention, suggesting that the development of prospective memory across early school years may be modulated by whether a cue requires overt monitoring beyond the immediate attentional context. Because whether a cue is in or outside of the center of attention might determine the amount of executive control needed in a prospective memory task, findings suggest that developing executive control resources may drive prospective memory development across primary school age.
\end{abstract} (c) 2013 Elsevier Inc. All rights reserved.

\footnotetext{
* Corresponding author.

E-mail address: matthias.kliegel@unige.ch (M. Kliegel).
} 


\section{Introduction}

The processes associated with the task of carrying out delayed intentions are referred to as prospective memory (Einstein \& McDaniel, 1990; Ellis, 1996; Ford, Driscoll, Shum, \& Macaulay, 2012; see Kliegel, McDaniel, \& Einstein, 2008, for an overview). Examples of prospective memory tasks in everyday life are remembering to pass on a message to a teacher when you next see her or to call the football coach at 12 o'clock. Conceptually, the successful completion of a prospective memory task requires the timely detection of the prospective cue and the self-initiated execution of the prospective action (prospective component associated with executive control processes; see, e.g., Einstein \& McDaniel, 1996; Kliegel, Altgassen, Hering, \& Rose, 2011; McDaniel \& Einstein, 2000) as well as the correct retrieval of the to-be-performed intention (retrospective component mostly associated with memory-related processes). Methodologically, the cue for the intended action is almost always embedded within ongoing activities, referred to as the ongoing task that needs to be interrupted before being able to switch to execute the prospective task. Hence, prospective memory has been described as a combination of memory and executive control processes (e.g., Ellis, 1996; Kliegel et al., 2011).

There are two main types of prospective memory tasks: event-based and time-based (Kvavilashvili \& Ellis, 1996). Event-based prospective memory tasks require an individual to initiate the intended action after the occurrence of an external event signaling the appropriate context for the execution (e.g., "remember to take the cake out of the oven when the timer rings"), whereas time-based tasks require an individual to remember to perform the intended action at a specific point in time or after a specified period of time has elapsed (e.g., "remember to feed the dog at 6 pm").

\section{Development of prospective memory during childhood: Current evidence}

The focus of the current study was on the development of event-based prospective memory, specifically on the developmental changes that occur over the primary school years. Although there is a vast body of research on prospective memory development across late adulthood (see Henry, MacLeod, Phillips, \& Crawford, 2004; Ihle, Hering, Mahy, Bisiacchi, \& Kliegel, in press; Kliegel, Jäger, \& Phillips, 2008, for meta-analytic overviews), surprisingly few studies have investigated the development of prospective memory during childhood (see Kvavilashvili, Kyle, \& Messer, 2008, for a review). This lack of research on prospective memory development is particularly concerning because prospective memory is a central and necessary skill to cope with the demands of children's everyday lives (Meacham, 1982; Winograd, 1988), especially in regard to the development of independence and autonomy during childhood (e.g., Kvavilashvili, Messer, \& Ebdon, 2001; Meacham, 1982). To date, only a handful of studies have examined event-based prospective memory during the late preschool to early school years, and these few studies have revealed somewhat contradictory results.

An early study examined age differences in event-based prospective memory across the late preschool and early school years by focusing on retrieval context (Kvavilashvili et al., 2001). In three experiments, 4-, 5-, and 7-year-old children were asked to name objects pictured on cards (ongoing task) and also to remember to put cards with animals on them into a box (prospective memory task). Ongoing task interruption was targeted as a possible mechanism underlying developmental differences in prospective memory performance. Therefore, task interruption was experimentally manipulated so that for some children the prospective cue appeared in the middle of the deck of cards (task interruption condition) and for other children the cue appeared at the end of the deck of cards after the ongoing task had been completed (no interruption condition). Task interruption resulted in worse prospective memory performance compared with the no interruption condition. A small age effect was revealed, with 7 -year-olds showing better prospective memory performance than 4 - and 5 -year-olds. Even in the face of minimal demands on task interruption (i.e., in the no interruption condition), older children outperformed younger children; hence, increasing ability to interrupt the ongoing task was not found to contribute to developmental change in prospective memory, and so the question of which processes underlie prospective memory development remains open. 
During recent years, several cognitive mechanisms behind the age-related increases in prospective memory have been proposed. Besides the important role of retrospective memory processes in prospective memory development (see Kerns, 2000; Smith, Bayen, \& Martin, 2010), gains in executive processes such as inhibitory control and working memory have been suggested to serve as developmental mechanisms underlying developmental growth in prospective memory. Several authors (including the current ones) have argued that executive processes may play an important role in the age effect in prospective memory via developing the ability to switch from one activity to another (shifting) during childhood (see, e.g., Kliegel, Mackinlay, \& Jäger, 2008).

Recent studies on preschoolers and young children, however, have revealed mixed evidence for the role of executive processes in event-based prospective memory (Atance \& Jackson, 2009; Ford et al., 2012; Mahy \& Moses, 2011; Wang, Kliegel, Liu, \& Yang, 2008). Whereas Ford and colleagues (2012) reported evidence for the independent predictive role of inhibitory control but not working memory in preschoolers' event-based prospective memory (although working memory and inhibitory control were positively correlated with prospective memory), Mahy and Moses (2011) found that working memory but not inhibitory control predicted event-based prospective memory in the same-age group. Similarly, Atance and Jackson (2009) found no association between event-based prospective memory and performance on two executive tasks (Tower of London and Delay of Gratification) in 3- to 5-yearold children after controlling for age. None of the above-mentioned studies explicitly examined executive processes as a developmental mechanism in that it did not explore whether age-related differences in event-based prospective memory could be explained by differences in executive abilities (e.g., by using a mediation analysis). However, Mahy and Moses (2011) found that the age effect on event-based prospective memory persisted after controlling for inhibitory control and working memory, suggesting that these two abilities were not entirely responsible for the age-related gains in prospective memory performance. Thus, at least in preschoolers and young children, the role of executive processes in age-related gains in prospective memory is uncertain.

Few studies have investigated the role of executive abilities in event-based prospective memory during middle childhood (7-13 years; but see Shum, Cross, Ford, \& Ownsworth, 2008; Yang, Chan, \& Shum, 2011). One such study found that event-based prospective memory was significantly predicted by verbal fluency, working memory, inhibition, and cognitive flexibility after controlling for age in 8- and 9-year-old and 12- and 13-year-old children (Shum et al., 2008). In contrast, time-based prospective memory has been shown to positively correlate with inhibition, visual working memory, and set shifting, and performance on measures of this construct increases between 7 and 12 years of age (Kerns, 2000). Similarly, Mackinlay, Kliegel, and Mäntylä (2009) showed that the majority of agerelated variance in 7- to 12 -year-olds' time-based prospective memory can be explained by planning and task-switching ability. However, other research has suggested that inhibition and updating, but not set shifting, are related to monitoring performance in a time-based prospective memory task in 8- to 12-year-old children (Mäntylä, Carelli, \& Forman, 2007). Taken together, the relation between executive abilities and time-based prospective memory is fairly well documented during middle childhood. Far fewer studies have examined the relation between executive control and event-based prospective memory. Moreover, all of the reviewed research during this middle childhood period except Shum and colleagues' (2008) study has been correlational in nature and has not experimentally manipulated factors thought to involve executive function. The current study attempted to take an experimental approach by systematically manipulating factors that have been argued to vary the level of executive demand, specifically those that affect the ability to switch flexibly between performing the ongoing task and detecting the target prospective memory cue.

Some support for the role of executive controlled processes in age-related increases in prospective memory is provided by a study with older children (Smith et al., 2010) that compared the relative importance of both components of prospective memory (retrospective vs. prospective components) and found descriptive age differences in prospective memory performance, with 10-year-olds outperforming 7-year-olds. Moreover, young adults had better prospective memory performance than either group of children. Analyses using multinomial models showed that differences between younger and older primary school children could be attributed to differences in the retrospective memory component but not in the prospective component (mostly measuring controlled attention in terms of target monitoring). In contrast, better performance of the adult group was related to differences in both the 
memory and controlled attention components. Smith and colleagues (2010) inferred that during early school years there might be less developmental growth with respect to the preparatory attentional control processes involved in detecting the prospective cue. Following this logic, age-related increases in retrospective memory, but not in attentional control processes, may underlie the observed age effect in prospective memory performance across the primary school years.

A different conclusion, however, was recently presented by Yang and colleagues (2011), who examined the development of prospective memory across several time- and event-based prospective memory tasks. Yang and colleagues found a developmental increase from 7 to 8 years of age and from 10 to 11 years of age. In addition, when performance was collapsed across all tasks, prospective memory was positively correlated with independent measures of working memory and inhibition, suggesting some involvement of executive functions in prospective memory in this age range. However, these correlations seemed to be driven partly by shared age-related variance because the composite score no longer correlated with the two executive measures after partialling out age. Hence, specific mechanisms driving the observed age effects on event-based prospective memory remain unclear.

Although most of the studies on early school-age children reveal at least some age differences (but differ in their conclusions on possible cognitive mechanisms), one study did not find age-related effects in 7- to 11-year-olds' prospective memory in a task that required children to pass a message to a research assistant as soon as they entered the room (Nigro, Senese, Natullo, \& Sergi, 2002). Overall prospective memory performance was quite high for this task and may have been supported by the very salient and socially relevant (human) cue in this task. Because no factors relevant to cue event detection were manipulated, this conclusion remains speculative and the role of cue salience remains to be tested directly.

\section{Interim conclusions}

Taken together, the few available studies on event-based prospective memory development across late preschool and primary school have targeted different age ranges and have failed to consistently identify age differences. Although Nigro and colleagues (2002) did not find age-related effects in a group of 7- to 11-year-olds, at least some evidence for developmental growth was revealed by Smith and colleagues (2010) and Yang and colleagues (2011). Moreover, heterogeneous results were obtained by Kvavilashvili and colleagues (2001), who reported better performance by 7-year-olds compared with 4- and 5-year-olds, yet 7-year-olds outperformed 5-year-olds in only two of three experiments. In contrast, studies of children during the late preschool, early primary school, and middle childhood periods reveal consistent evidence for developmental increases in prospective memory performance from 3 to 12 years of age (e.g., Atance \& Jackson, 2009; Ford et al., 2012; Kerns, 2000; Mahy \& Moses, 2011; Shum et al., 2008).

Cross-referencing studies, these mixed findings on the effect of age may be the result of using different tasks, which likely varied on multiple dimensions that are critical for prospective memory performance, and this may have affected the probability of finding an overall age effect. Consequently, a series of studies using the same prospective memory task and systematically varying dimensions of such a task would help to clarify the heterogeneous findings that exist. Furthermore, such studies may help to identify common underlying developmental mechanisms. Besides the mixed results on age effects, so far no clear picture on possible developmental mechanisms for developmental increases in event-based prospective memory has emerged. Smith and colleagues (2010) argued in favor of memory-related processes, whereas others have suggested executive functions as driving age effects (Kvavilashvili et al., 2001; Yang et al., 2011), although these latter studies do not rule out contributions of memory processes because they were not examined directly. In contrast, research on preschool-age and early school-age children has revealed contradictory results concerning the involvement of inhibitory control, working memory, and planning in the development of event-based prospective memory (e.g., Atance \& Jackson, 2009; Ford et al., 2012; Mahy \& Moses, 2011). Furthermore, much of the research on school-age children has focused on the relation between various executive functions and time-based prospective memory rather than event-based prospective memory, and (importantly) these studies used mainly correlational methods. The current study, therefore, attempted to examine 
the effect of age and executive processes on older children's event-based prospective memory via experimental manipulations of factors thought to influence executive demand.

\section{The current study}

The aim of the current study was to help clarify the heterogeneous pattern of findings by providing a systematic examination of the role of three retrieval-based factors to better understand age differences in event-based prospective memory in early school-age children. This approach was informed conceptually by the multiprocess theory (McDaniel \& Einstein, 2000) and the preparatory attentional and memory (PAM) model (Smith, 2003; Smith \& Bayen, 2004), which suggest that specific factors that more or less rely on executive controlled processes will contribute to successful event-based prospective memory retrieval. In the current study, a systematic evaluation of key factors suggested by or in the context of these models of prospective memory was carried out to examine possible developmental mechanisms associated with age effects across early childhood.

The basic assumption of both frameworks is that prospective memory performance may involve more or less top-down processes (e.g., executive control processes) and that their relative prominence varies systematically as a function of specific contextual features of the prospective task. Importantly, it is well documented that controlled processing resources such as executive control are subject to marked developmental changes across the early school years (e.g., Gathercole, Pickering, Ambridge, \& Wearing, 2004; Schneider \& Bjorklund, 1998; Schneider \& Pressley, 1997; Welsh, Pennington, \& Groisser, 1991; Zelazo, Carlson, \& Kesek, 2008; Zelazo, Müller, \& Frye, 2003). In particular, working memory, inhibitory control, and task switching become more efficient (see Best, Miller, \& Jones, 2009, for an overview; Carlson, 2005), and these developmental changes may contribute to the development of event-based prospective memory during childhood. If growing executive control capacities indeed underlie, or at least contribute to, developmental increases in prospective memory, then performance differences between younger children (who usually have poorer executive control) and older children (who usually have better executive control) should be larger in prospective tasks that are executively demanding. Consequently, any manipulations that reduce demands placed on controlled processes may be expected to attenuate or even eliminate age differences across early school years. Three main retrieval-associated factors are predicted to vary executive processing demands: (a) ongoing task absorption, (b) cue salience, and (c) cue centrality.

\section{Ongoing task absorption}

Prospective memory models suggest that the more demanding, engaging, or absorbing the ongoing task, the more dual-task control and monitoring resources will be required to detect the prospective cue; for example, while being engaged in conversation with a friend, higher strategic monitoring may be necessary to maintain the intention to buy bread for dinner when you pass the bakery compared with when walking alone. Thus, higher ongoing task absorption should lead to larger age-related differences. This prediction was tested in Experiment 1.

\section{Cue salience}

Perceptually salient prospective memory cues are predicted to support performance by eliciting a kind of orienting response; for example, while passing a mailbox, a large colorful box should be a more salient cue to mail a letter than a small box that is camouflaged by the colors of the surrounding buildings (see McDaniel \& Einstein, 2000). Thus, the less salient the prospective memory task cue, the more controlled monitoring may be required, which should also result in larger age effects. Alternatively, the more salient a cue, the more likely bottom-up processes such as associative processes may trigger prospective memory execution. This prediction was tested in Experiment 2. 


\section{Cue centrality}

Finally, whether the prospective memory cue is in or outside of the center of attention affects the processing required for the ongoing task and may affect prospective memory performance. Prospective memory cues in the center of attention are those in which the cues appear within the focus of attention while completing the ongoing task that do not require additional search or monitoring processes for detection (Guynn, 2003). In this case, it can be assumed that the prospective memory cues are easier to detect without activating additional search processes for the prospective memory cues. In contrast, prospective memory tasks with cues outside of the center of attention are those in which the prospective memory cue is outside the scope of attentional focus of the ongoing activity such as driving home in bad traffic while needing to remember to stop at the grocery store that is just off your route (see McDaniel, Einstein, \& Rendell, 2008, for this example). Therefore, periodically attention needs to be shifted in order to search for the appearance of the prospective memory cues. Prospective memory cues are often outside of the center of attention in time-based prospective memory tasks due to the need to monitor a clock behind one's back. In outside of the center of attention tasks, prospective remembering is thought to require more controlled resources in order to carry out extra monitoring for the cue to perform an intended action; in other words, attention must be divided between the ongoing task and monitoring for the prospective memory cues. These additional controlled processes necessary for detection of the outside of the center of attention target cues should lead to more pronounced age effects in prospective memory tasks. This prediction was tested in Experiment 3.

To the best of our knowledge, the current study is the first to systematically examine a comprehensive set of predictions derived from the current prospective memory frameworks for possible age differences across early school years as well as developmental mechanisms that may underlie those age differences. Importantly, across all three experiments (one for each retrieval-associated factor), the same general experimental procedure was used. Therefore, any differences between studies cannot be attributed to differences in specific measures or general procedure.

\section{Experiment 1}

In Experiment 1, the effect of ongoing task absorption on age differences in event-based prospective memory performance during the primary school years was examined.

\section{Method}

\section{Participants}

Participants were 66 children recruited from local primary schools. The younger age group consisted of 33 children (18 girls and 15 boys) who were 6 or 7 years of age ( $M=6.88 \pm 0.33$ years), and the 33 children (14 girls and 19 boys) in the older age group were 9 or 10 years of age $(M=9.67 \pm 0.54$ years). No significant gender differences emerged between age groups. All children scored within \pm 1 standard deviation on standardized measures of fluid and crystallized intelligence (see "General ability" subsection in "Materials and procedures" section and Table 1 for means by age group).

\section{Materials and procedure}

Prospective memory task. Following the recommendations of Kvavilashvili and colleagues (2008), the experimental procedure used in this study (the Dresden Cruiser; see Voigt, Aberle, Schönfeld, \& Kliegel, 2011, for a time-based version) was based on a driving game scenario initially introduced by Kerns (2000) (see also Kerns \& Price, 2001). In this game, the ongoing task was to drive a vehicle on a road without crashing into other cars, and the prospective memory task was to remember to refuel the car before it ran out of gas. Specifically, children were engaged in driving a car on a twodimensional road that was displayed vertically on the monitor. The road consisted of three parallel lanes, with other vehicles driving on the road in the same direction. The car was controlled by a gamepad (Thrustmaster FireStorm Digital 3 Gamepad). Children were able to maneuver on the horizontal 
Table 1

Means and standard deviations for fluid and crystallized intelligence by age group in each experiment and whether differences between groups are significant.

\begin{tabular}{llllll}
\hline & & Age group & & \multirow{2}{*}{$t$ value } & \multirow{2}{*}{$d$} \\
\cline { 3 - 5 } & & 6- and 7-year-olds & 9- and 10-year-olds & & \\
\hline \multirow{2}{*}{ Experiment 1 } & Fluid intelligence & $10.48(3.25)$ & $11.15(3.46)$ & 0.80 & 0.21 \\
& Crystallized intelligence & $10.76(2.88)$ & $12.61(2.94)$ & $2.58^{*}$ & 0.63 \\
Experiment 2 & Fluid intelligence & $9.27(2.97)$ & $11.00(3.34)$ & $2.35^{*}$ & 0.55 \\
& Crystallized intelligence & $10.27(3.02)$ & $10.54(2.80)$ & 0.40 & 0.01 \\
Experiment 3 & Fluid intelligence & $10.79(2.11)$ & $12.87(2.68)$ & $3.81^{* *}$ & 0.86 \\
& Crystallized intelligence & $11.33(2.67)$ & $11.67(2.62)$ & 0.56 & 0.13 \\
\hline
\end{tabular}

Note. Standard deviations are in parentheses. Cohen (1988) defined effect sizes of 0.20 as small, 0.50 as medium, and 0.80 as large.

${ }^{*} p<.05$.

** $p<.01$

axis (left-right) but not on the vertical axis (forward-backward). The aim of the ongoing task was to get as many points as possible by avoiding hitting other cars. A prospective memory task was embedded in this ongoing driving task; children needed to remember to refuel the car. It was only possible to refuel when one-fourth or less fuel was left in the tank. In contrast to Kerns (2000) and Voigt and colleagues (2011), where children needed to monitor the fuel gauge as a time equivalent, in all three experiments of the current study children were cued by specific events that the tank was one-fourth full and were instructed to remember to press a button on the gamepad to refuel whenever that particular event occurred. After pressing the button, a fuel gauge appeared for $4 \mathrm{~s}$ in the left lower corner of the screen, indicating that the refuel was successful. The tank was refilled automatically when the car ran out of gas, but no fuel gauge appeared. Whenever participants performed the prospective memory task successfully, extra points were added to the score. Without refilling, the car ran out of gas after $1 \mathrm{~min}$; after $45 \mathrm{~s}$, the tank could be filled within a time window of $15 \mathrm{~s}$. The duration of one game cycle was $4 \mathrm{~min}$. Each child played two cycles of the game (within-participants variation of ongoing task absorption; see below), with a total of eight prospective memory cues appearing. Therefore, children were given prospective memory performance accuracy scores based on their performance on the four prospective memory trials in each ongoing task absorption condition.

General ability. To assess crystallized intelligence, the Information subscale of the German version of the Wechsler Intelligence Scale for Children (HAWIK-IV; Petermann \& Petermann, 2008) was used. Children were asked general knowledge questions of increasing difficulty. The Block Design subscale of the HAWIK-IV was used to assess fluid intelligence. In this subscale, participants needed to manually replicate abstract figures using small multicolored plastic blocks.

Procedure. The general procedure followed Kvavilashvili and colleagues' (2008) suggestion to separate the prospective memory procedure into several short subcomponents. Two blocks of 4 min were administered in which the experimental factor of ongoing task absorption was varied within participants (see Fig. 1). Block order was counterbalanced. First, children were told about the ongoing task. When children could repeat the instructions for this task accurately, they played a 30-s practice trial to familiarize themselves with the game. In the practice trial, no refueling was required. If children hit cars on purpose, they were asked to repeat the instructions and it was emphasized that they would lose points by hitting other vehicles. Then, children were given the prospective memory task instructions verbally without any examples of the prospective memory cues and were not given an opportunity to practice the prospective memory task. The prospective cue in Experiment 1 was a change in the color of flowers passing by at the side of the road. Flowers were usually displayed in soft pink, but the refuel cue was a flowerpot with yellow flowers that appeared on both sides of the road. Pilot work had revealed that an overlearned single event (e.g., a gas station) produced ceiling effects even among the younger children and that children readily accepted the flowerpot cue as a normal part of the game. Again, children were required to show understanding of task instructions by verbal recall of the game 


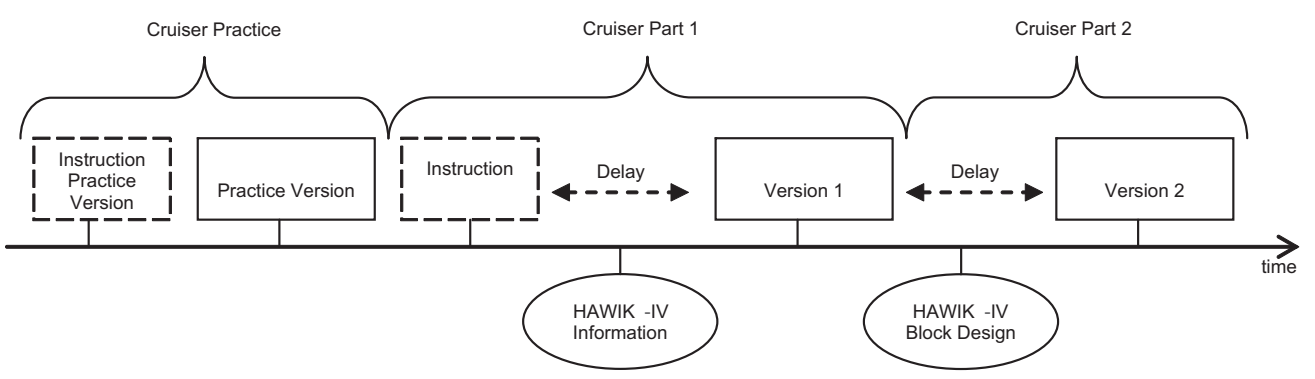

Fig. 1. General procedure of Experiment 1. WAIS, Wechsler Intelligence Scale for Children (HAWIK-IV).

rules. However, to introduce a delay between task instructions and subsequent execution of the prospective memory task (see Ellis \& Kvavilashvili, 2000), children were told that they would first play another game, the Information scale of the HAWIK-IV. After completing the Information task, children were asked to play the first round of the cruiser without further reminding of the need to refuel.

To manipulate ongoing task absorption in Experiment 1, children played either a less demanding version with 15 other cars appearing on the road per minute (low task absorption) or a more demanding version with 35 cars per minute first (high task absorption). Thereafter, another delay was implemented as children completed the Block Design task of the HAWIK-IV. When finished with the Block Design task, children played the second round of the cruiser game in which they received the other experimental condition that they had not received in the first block. Afterward, retrospective memory for task instruction was tested; all children (in all three experiments reported) were able to recall the instructions for the prospective memory task. Finally, children received a voucher ( 5 euros) to a local toy store. The procedure lasted approximately $40 \mathrm{~min}$ in total. Children were tested individually.

\section{Results}

Ongoing task performance (manipulation check)

The number of times children crashed into other cars was used as an indicator of ongoing task performance (see Voigt et al., 2011; Table 2). A mixed $2 \times 2$ analysis of variance (ANOVA) with the withinparticipants factor ongoing task absorption (high or low) and the between-participants factor age group (younger or older children) was conducted on ongoing task performance. The analysis revealed a main effect of ongoing task absorption, indicating more car crashes in the high-absorption condition than in the low-absorption condition, $F(1,64)=925.65, M S E=88.85, p<.001, \eta_{\mathrm{p}}{ }^{2}=.94$. In addition, there was a main effect of age group, indicating more car crashes in the younger age group than in the older age group, $F(1,64)=18.96, M S E=262.75, p<.001, \eta_{\mathrm{p}}{ }^{2}=.23$. Moreover, an interaction emerged between ongoing task absorption and age, $F(1,64)=4.79, M S E=88.85, p=.032, \eta_{\mathrm{p}}{ }^{2}=.07$.

\section{Prospective memory performance}

A mixed $2 \times 2$ ANOVA was conducted with the within-participants factor ongoing task absorption (high or low) and the between-participants factor age group (younger or older children) on prospec-

\section{Table 2}

Ongoing task performance (car crashes) in all experiments.

\begin{tabular}{llll}
\hline & Condition & $\begin{array}{l}\text { Younger children } \\
{[M(S D)]}\end{array}$ & $\begin{array}{l}\text { Older children } \\
{[M(S D)]}\end{array}$ \\
\hline Experiment 1 & Low absorption & $20.3(10.1)$ & $11.6(6.9)$ \\
& High absorption & $73.8(17.5)$ & $57.9(15.7)$ \\
Experiment 2 & Low salience & $72.5(17.4)$ & $57.1(16.9)$ \\
& High salience & $77.5(19.3)$ & $58.8(22.8)$ \\
Experiment 3 & In the center of attention & $83.3(21.2)$ & $60.8(15.8)$ \\
& Outside of the center of attention & $81.9(17.7)$ & $65.5(17.3)$ \\
\hline
\end{tabular}


tive memory performance. The analysis revealed a main effect of ongoing task absorption, $F(1,64)=14.82, M S E=0.05, p<.001, \eta_{\mathrm{p}}{ }^{2}=.19$, as well as a main effect of age group, $F(1,64)=11.59$, $M S E=0.17, p<.001, \eta_{\mathrm{p}}{ }^{2}=.15$, but no interaction effect $(F \leqslant 1)$ (see Fig. 2 ). Older children remembered to refill the car more often than younger children, and children remembered to refuel the car more often in tasks with low ongoing task absorption compared with high ongoing task absorption.

To ensure that ongoing task performance was not responsible for the effect of age on prospective memory performance, an analysis of covariance (ANCOVA) was conducted on the age effect on prospective memory performance separately for the low- and high-absorption conditions with ongoing task performance as a covariate. After covarying ongoing task performance, age differences remained significant in the low-absorption condition, $F(1,63)=25.32, M S E=3.11, p=.006, \eta_{\mathrm{p}}{ }^{2}=.12$, and remained marginally significant in the high-absorption condition, $F(1,63)=9.42, M S E=2.59, p=.061$, $\eta_{\mathrm{p}}{ }^{2}=.06$, with older children outperforming younger children. This suggests that ongoing task performance was not responsible for age effects.

\section{Discussion}

Taken together, results clearly showed age differences in prospective memory across early school years, in contrast to an earlier finding of no performance differences in this age range (Nigro et al., 2002). In addition, findings revealed that ongoing task absorption affected children's prospective memory performance such that better prospective memory occurred in the less absorbing ongoing task compared with the more absorbing ongoing task. However, in terms of possible developmental mechanisms underlying this age effect, Experiment 1 provided no support for the prediction that higher levels of ongoing task absorption (which supposedly should require more controlled monitoring resources for detecting the prospective cue) would amplify the reported age effect. In fact, ongoing task absorption affected both younger and older children's prospective memory equally; therefore, controlled processes that can be assumed to be associated with the effects of task absorption (e.g., dual-task control) appeared not to contribute to the developmental differences observed given that the age difference in prospective memory remained significant even after controlling for ongoing task performance.

\section{Experiment 2}

In Experiment 2, a second factor thought to affect the extent to which executive controlled processes are recruited was examined. Specifically, the effect of perceptual cue salience on age differences in event-based prospective memory performance across primary school age was tested.

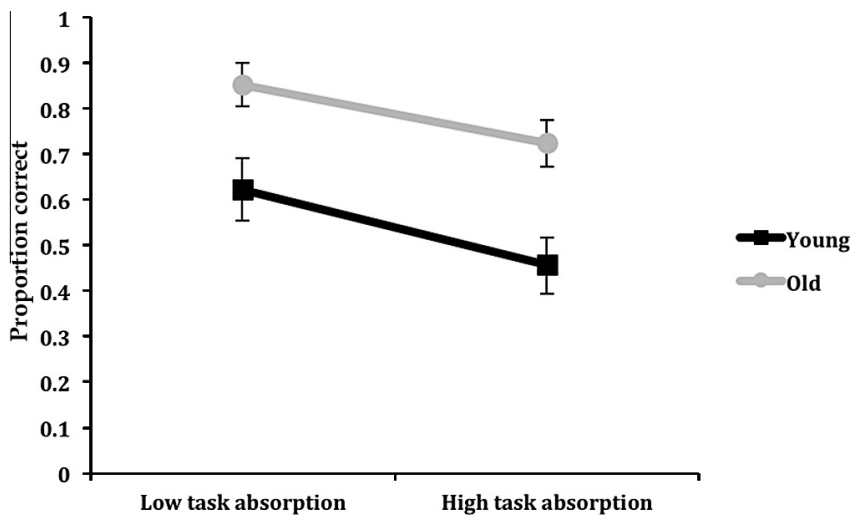

Fig. 2. Prospective memory performance in Experiment 1. Error bars represent 1 standard error of the mean. 
Method

\section{Participants}

Participants were 76 children recruited from local primary schools. The younger age group consisted of 37 children ( 14 girls and 23 boys) who were 6 or 7 years of age ( $M=7.25 \pm 0.49$ years), and the 39 children (18 girls and 21 boys) in the older age group were 9 or 10 years of age ( $M=9.73 \pm 0.51$ years). No significant gender differences emerged between the age groups. All children scored within \pm 1 standard deviation on standardized measures of fluid and crystallized intelligence.

\section{Materials and procedure}

Materials were identical to those in Experiment 1, but this time cue salience was varied within participants in the driving game instead of ongoing task absorption. In the low-salience condition, one flowerpot on each side of the road occurred with yellow flowers, in contrast to standard pink flowers. In the high-salience condition, several yellow flowerpots occurred. Ongoing task absorption was held constant at high difficulty (i.e., 35 cars/min) because this was shown to be more challenging and engaging for children in Experiment 1.

\section{Results}

\section{Ongoing task performance}

Ongoing performance was analyzed using a $2 \times 2$ mixed-factorial ANOVA that included the withinparticipants factor cue salience (high or low) and the between-participants factor age (younger or older children) (see Table 2). Younger children hit other cars more often than older children, $F(1,74)=17.87, M S E=614.72, p<.001, \eta_{\mathrm{p}}{ }^{2}=.20$, but there was a trend level effect of cue salience on ongoing task performance, $F(1,74)=3.35, M S E=126.92, p=.071, \eta_{\mathrm{p}}{ }^{2}=.04$, and no interaction between age and cue salience $(F \leqslant 1)$.

\section{Prospective memory performance}

Prospective memory performance was analyzed using a $2 \times 2$ mixed-factorial ANOVA that included the within-participants factor cue salience (high or low) and the between-participants factor age (younger or older children). Prospective memory performance was higher when a high-salience cue was presented compared with a low-salience cue, $F(1,73)=9.63, M S E=0.06, p=.003, \eta_{\mathrm{p}}{ }^{2}=.12$. In addition, performance improved with age, $F(1,73)=13.91, M S E=0.16, p<.001, \eta_{\mathrm{p}}{ }^{2}=.16$. Yet, no interaction between age and cue salience emerged, $F(1,73)=1.36, M S E=0.06, p=.248, \eta_{\mathrm{p}}{ }^{2}=.02$ (see Fig. 3 ).

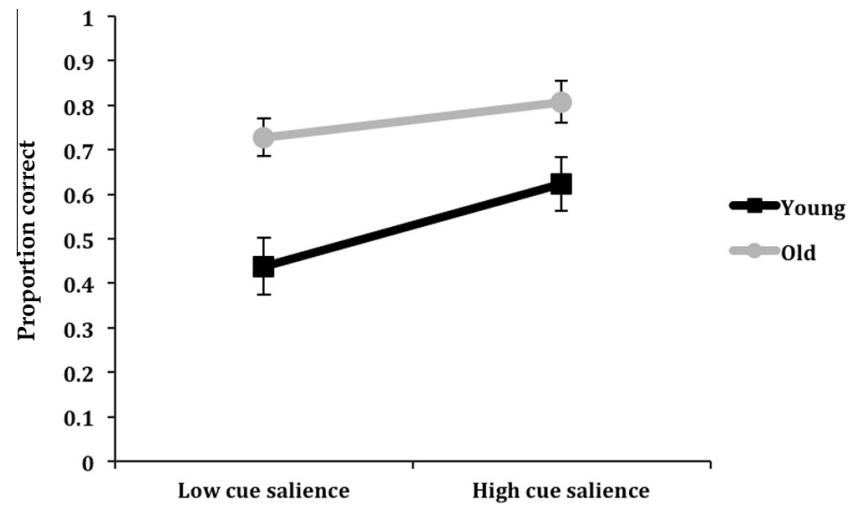

Fig. 3. Prospective memory performance in Experiment 2. Error bars represent 1 standard error of the mean. 
Because ongoing task absorption was not manipulated directly in this experiment, age differences in ongoing task performance were considered. To test the influence of ongoing task performance on the age effect in prospective remembering, an ANCOVA that controlled for ongoing task performance was applied. The results still revealed an effect of age, $F(1,72)=10.19, M S E=0.16, p=.002, \eta_{\mathrm{p}}{ }^{2}=.12$, as well as an effect of cue salience, $F(1,72)=5.76, M S E=0.06, p=.019, \eta_{\mathrm{p}}{ }^{2}=.07$, but an interaction emerged at the level of a trend, $F(1,73)=3.16, M S E=0.06, p=.080, \eta_{\mathrm{p}}{ }^{2}=.04$. Follow-up analyses revealed that there was a significant effect of age in the low-salience condition, $F(1,72)=14.37$, $M S E=0.11, p<.001, \eta_{\mathrm{p}}{ }^{2}=.17$, with older children outperforming younger children, whereas there was a smaller trend-level effect of age in the high-salience condition, $F(1,73)=2.89, M S E=0.11$, $p=.093, \eta_{\mathrm{p}}{ }^{2}=.04$, after controlling for ongoing task performance.

\section{Discussion}

These results replicate the finding of age differences found in Experiment 1, even identifying an effect size of comparable magnitude $\left(\eta_{\mathrm{p}}{ }^{2}\right.$ of .15 in Experiment 1 and .16 in Experiment 2 ), and (importantly) age differences in prospective performance could not be attributed to age differences in ongoing task performance. Thus, these data clearly support the notion of event-based prospective memory development across primary school age. In addition, the results indicate that cue salience may indeed affect event-based prospective memory performance in children. Yet, analyses resulted in no clear interaction effect, suggesting that less controlled associative processes related to the effects of high cue salience might not eliminate the developmental increases observed in prospective memory performance during early school years, although those associative processes can be assumed to be effective even during relatively early stages of cognitive development. Importantly, after covarying ongoing task performance, the interaction between age and cue salience became significant at the level of a trend, suggesting that perhaps with a larger sample size a pattern of high-salience cues helping to boost especially younger children's prospective memory performance may have emerged; yet, lowsalience cues, which presumably require more executive effort, would likely still produce age differences in performance, and with larger power also the trend level age effect on high-salience cues is likely to emerge in the follow up analyses, still suggesting persisting age differences even for salient cues.

\section{Experiment 3}

In Experiment 3, we examined whether cue centrality (cues presented in the center of attention versus outside of the center of attention) had an effect on prospective memory retrieval. In addition, we tested whether cue centrality might moderate age differences in event-based prospective memory across the early school years.

\section{Method}

\section{Participants}

Participants were 78 children recruited from local primary schools. The younger age group consisted of 39 children ( 12 girls and 27 boys) who were 6 or 7 years of age ( $M=6.68 \pm 0.47$ years), and the 39 children (21 girls and 18 boys) in the older age group were 9 or 10 years of age ( $M=9.50 \pm 0.51$ years). No significant gender differences emerged between age groups; however, because gender distribution was relatively uneven, we tested for gender effects in the variables of interest, but none of the variables analyzed below was affected by gender. All children scored within \pm 1 standard deviation on standardized measures of fluid and crystallized intelligence.

\section{Materials and procedure}

Materials were identical to those in Experiments 1 and 2 except for the within-participants manipulation of the cue in the center of attention or outside of the center of attention in the driving game. In 
the outside of the center of attention condition, the prospective memory cue was the yellow flowerpot outside of the road (as in Experiments 1 and 2; low salience). For the in the center of attention condition, the cue was presented in the focus of the attention required for the ongoing task (i.e., trying to avoid hitting other cars). Here, the prospective memory cue was a yellow car that needed to be overtaken during the process of the driving activity (no other cars were yellow), and children needed to fill the gas tank when they saw the yellow car. Ongoing task absorption was again held constant at high difficulty (i.e., 35 cars $/ \mathrm{min}$ ), and low cue salience (i.e., one flower pot at each side of the road) was used to ensure that the task remained challenging and engaging for children.

\section{Results}

\section{Ongoing task performance}

Ongoing performance was analyzed using a $2 \times 2$ mixed-factorial ANOVA that included the withinparticipants factor cue centrality (in the center of attention or outside of the center of attention) and the between-participants factor age (younger or older children) (Table 2). Younger children hit other cars more often than older children, $F(1,76)=29.03, M S E=508.18, p<.001, \eta_{\mathrm{p}}{ }^{2}=.28$, but there was no difference in ongoing task performance between the two cue centrality conditions, $F(1,76)=0.77$, $M S E=146.77, p=.382, \eta_{\mathrm{p}}{ }^{2}=.01$, and no interaction, $F(1,76)=2.45, M S E=146.77, p=.121, \eta_{\mathrm{p}}{ }^{2}=.03$.

\section{Prospective memory performance}

Prospective memory performance was analyzed using a $2 \times 2$ mixed-factorial ANOVA that included the within-participants factor cue centrality (in the center of attention or outside of the center of attention) and the between-participants factor age (younger or older children). Prospective memory performance was better with a cue in the center of attention than with a cue outside of the center of attention, $F(1,76)=26.91, M S E=771.93, p<.001, \eta_{\mathrm{p}}{ }^{2}=.26$. In addition, performance improved with age, $F(1,76)=12.12, M S E=1564.64, p<.001, \eta_{\mathrm{p}}{ }^{2}=.14$. Moreover, an interaction between age and cue centrality emerged that just missed the conventional level of significance, $F(1,76)=3.84$, $M S E=771.93, p=.054, \eta_{\mathrm{p}}{ }^{2}=.05$ (see Fig. 4). Planned comparisons revealed that this marginal interaction was due to age differences being significant for the cues outside of the center of attention $\left(p<.001, \eta_{\mathrm{p}}{ }^{2}=.17\right)$ but not for the cues in the center of attention $\left(p=.084, \eta_{\mathrm{p}}{ }^{2}=.04\right)$. Strikingly, the age difference was more than four times larger in the outside of the center of attention condition than in the center of attention condition as indexed by effect size.

Because ongoing task performance differed between age groups, age differences in ongoing task performance were also considered. To test the influence of ongoing task performance on the age effect in prospective remembering, an ANCOVA was applied covarying ongoing task performance. The results still revealed a main effect of cue centrality, $F(1,75)=6.60, M S E=759.54, p=.012, \eta_{\mathrm{p}}{ }^{2}=.08$, but only a marginal age effect, $F(1,75)=3.59, M S E=1492.60, p=.062, \eta_{\mathrm{p}}{ }^{2}=.05$. However, an even

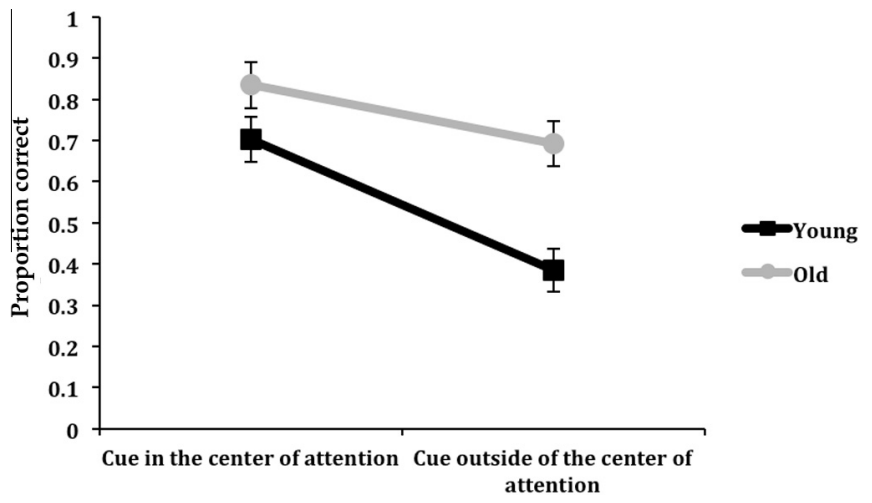

Fig. 4. Prospective memory performance in Experiment 3. Error bars represent 1 standard error of the mean. 
stronger interaction effect was revealed, $F(1,75)=6.10, M S E=759.54, p=.016, \eta_{\mathrm{p}}{ }^{2}=.08$. Planned comparisons covarying ongoing task performance showed that this interaction was again due to reliable age differences in the cues presented outside of the center of attention $\left(p<.001, \eta_{\mathrm{p}}{ }^{2}=.13\right)$ but an absence of an age differences for cues in the center of attention $\left(p=.754, \eta_{\mathrm{p}}{ }^{2}<.01\right)$ (see Fig. 5 for estimated means).

\section{Discussion}

Three main findings emerged from Experiment 3. First, data replicated the general age difference found in Experiments 1 and 2 (again with a comparable effect size of $\eta_{\mathrm{p}}{ }^{2}=.14$ ). Second, cue centrality was clearly identified as a variable affecting children's prospective memory performance. This main effect was approximately twice as large as the age effect identified and supports our prediction that whether the cue is in the center of attention or outside of the center of attention may be a key factor in determining prospective memory retrieval. Third, and most important from a developmental perspective, an age by cue centrality interaction was revealed, indicating that prospective memory performance of younger children (compared with older children) was much more negatively affected by a cues outside of the center of attention compared with cues in the center of attention. In fact, planned comparisons further exploring the interaction showed that the age-related effects on prospective memory emerged only in the outside of the center of attention condition (and not in the center of attention condition). Interestingly, younger children performed nearly as well as older children on the in the center of attention cue condition after covarying ongoing task performance, suggesting that cues in the center of attention may boost prospective memory performance in younger children to the level of older children.

Several possibilities exist to explain this age difference in prospective memory performance for cues outside of the center of attention. First, it is possible that the results reflect the effect of shifting visual attention (cognitive control account). Older children likely have superior executive control, allowing them to shift their attention to a cue outside of the center of attention in order to detect the prospective memory cue. It is likely that older children use proactive control in order to detect the cues outside of the center of attention. In contrast, younger children may struggle with shifting attention away from the ongoing task due to their relatively immature executive abilities and, therefore, must rely on reactive control that is not sufficient to detect cues outside of the center of attention. Both age groups performed similarly with cues in the center of attention because detection of these prospective memory cues presumably imposes fewer demands on executive control operations given that shifting one's attentional focus is not necessary.

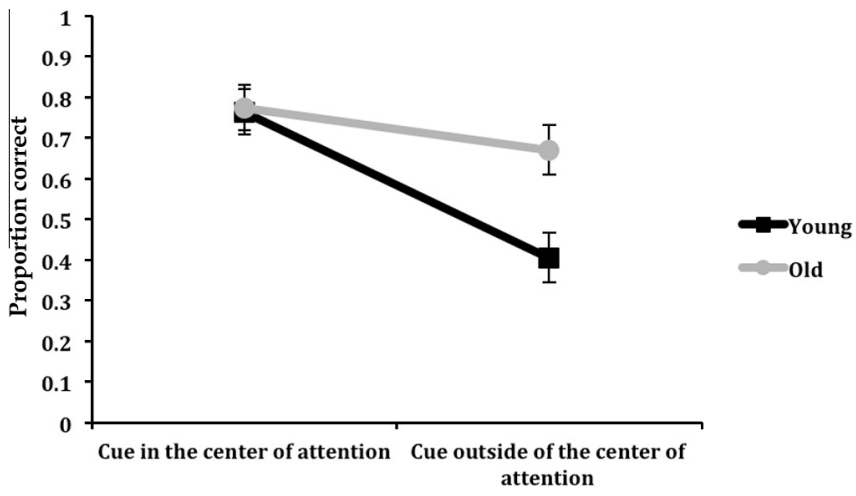

Fig. 5. Prospective memory performance after covarying ongoing task performance in Experiment 3. Error bars represent 1 standard error of the mean. 
Second, according to a metacognitive account, it is possible that older children had a more sophisticated checking strategy than younger children, allowing them to detect cues outside of the center of attention more frequently than younger children. Because detection of cues in the center of attention does not require a complex strategy, age differences would not be expected, whereas the cues outside of the center of attention require regular checking and perhaps awareness that checking will help performance on the task.

Finally, it is possible that differences in encoding the prospective memory instruction may account for the differences between younger and older children in their prospective memory performance for cues outside of the center of attention. Brewer and Marsh (2010) showed that differences in episodic encoding of the instruction resulted in differences in adults' prospective memory performance. In the current experiments, children's basic understanding of the prospective memory instruction was confirmed; however, it is possible that there were age-related differences in the depth of encoding, with older children having superior encoding. This alternative seems somewhat less plausible because one might expect older children to outperform younger children on prospective memory cues presented in the center of attention as well given differences in initial encoding.

Given these three possibilities, it will be important for future research to use mediational analyses and designs that can speak to the mechanism behind the age by cue centrality interaction. Future studies could investigate the relative impact of shifting attention, sophistication of checking strategies, or encoding depth to further examine what leads to larger age effects in prospective memory in response to a cue outside of central attention.

One limitation of Experiment 3 was that cue centrality was manipulated within a prospective memory task that had high ongoing task absorption and low cue salience, resulting in a fairly difficult task; however, children's prospective memory performance did not reach floor levels, and even young children in the cue outside of the center of attention condition successfully detected nearly half of the prospective memory cues.

\section{General discussion}

The current study provides the first systematic experimental evaluation of three retrieval-based factors that potentially contribute to age differences in event-based prospective memory function in young school-age children. Findings may help to clarify heterogeneous findings on developmental growth in this age range and argue for an important role of executive processes as possible mechanisms underlying developmental increases in prospective memory.

The first notable finding was that age differences in prospective memory performance were identified across all three experiments using one common and comparable prospective memory paradigm. Thus, an age-appropriate task of general interest for children was used, possible ceiling effects in the older group were eliminated, age differences in the ongoing task performance were considered, and retrospective memory for task instructions was confirmed. With each of these key methodological considerations in place, the current study provides strong evidence that 9- and 10-year-old children remember to execute delayed intentions better than 6 - and 7-year-old children. Thus, the current work identified a systematic developmental growth in prospective memory functioning during early school years and, therefore, contradicts results of prior studies that failed to identify age differences in prospective memory in this age range (see, e.g., Nigro et al., 2002). Prior studies have used a wide range of prospective memory paradigms that may have differed in multiple task features that are critical for prospective memory performance, and consequently the probability of finding age effects may have differed between studies.

Therefore, the current study was designed to systematically manipulate retrieval-based factors. As noted, the rationale for the specific manipulations was based on the predictions of the PAM model (Smith, 2003; Smith \& Bayen, 2004) and the multiprocess theory (McDaniel \& Einstein, 2000). These models propose that event-based prospective remembering can be supported by controlled processes such as monitoring the environment for the presence of the prospective cue or more associative retrieval processes. Consistent with these models, the provision of a less complex ongoing task (Experiment 1), higher cue salience (Experiment 2), and cues appearing in the center of attention (Experiment 3) 
were each associated with better prospective memory performance in both age groups. Therefore, these data have important conceptual and practical implications in identifying specific ways by which prospective memory may be optimized in school-age children. Moreover, they provide strong empirical evidence that the assumptions about the important role of controlled processes in prospective memory performance apply to early school-age children.

Important for conceptual advancement of the young field of children's prospective memory development, with respect to underlying developmental mechanisms, is that age differences were affected by only one of three retrieval-based factors. Cue centrality modulated developmental increases in prospective memory during early school years, with age effects in Experiment 3 being restricted to the outside of the center of attention (compared with in the center of attention) cue condition. Indeed, the absolute magnitude of the age effect in the outside of the center of attention condition was four times larger than in the in the center of attention condition, implying that age effects during early childhood may be dependent on whether a cue is in the center of attention or outside of the center of attention in the prospective retrieval context (see Scullin, McDaniel, Shelton, \& Lee, 2010). However, neither the complexity of the ongoing task nor cue salience interacted with age (although there was a trend toward an interaction after controlling for ongoing task performance) and, consequently, did not contribute to the developmental differences observed (see Rendell, Vella, Kliegel, \& Terrett, 2009, for similar results in a delay-execute paradigm in preschoolers).

One possibility for these differential effects is that the three retrieval-based factors vary in their demand for strategic and controlled processes. In this regard, it is of note that in the context of cognitive aging, it has been suggested that the location of the cue within or outside of focal attention may be the most important determinant of the operation of controlled processes (Kliegel, Jäger, \& Phillips, 2008; McDaniel \& Einstein, 2000; Scullin, McDaniel, \& Einstein, 2010; Scullin, McDaniel, Shelton, \& Lee, 2010). In fact, Einstein and McDaniel (2005) stated, "Whether or not there are [adult] age differences will depend on whether the prospective memory task uses nonfocal or focal target events" (p. 289).

Although the current manipulation did not vary cue focality, detecting the color of the flowerpots at the side of the road did not overlap at all with the main activities involved with avoiding cars during the ongoing task, whereas detecting the color of a car did to some extent converge with the activities involved in avoiding cars (here, it was not necessary to periodically divert attentional resources away from the main activity). Thus, with respect to the location of cue in the center of attention or outside of the center of attention, the current study appears to suggest that there may be similar developmental mechanisms that contribute to age differences in prospective memory from childhood across adolescence into old adulthood.

Although we recognize that there are several potential mechanisms by which cue centrality has an impact on children's prospective memory performance, we believe that attention shifting likely accounts for the age differences when there is a cue in the center of attention or outside of the center of attention. Older children may be able to shift attention more flexibly between the ongoing task and monitoring for a target outside of the center of attention, whereas younger children may have struggled with this switching, resulting in the detection of fewer prospective memory cues.

Another possibility that would be consistent with previous work is that mechanisms underlying gains in prospective memory function during this developmental period depend more heavily on the retrospective memory component compared with the prospective memory component (see Kerns, 2000; Smith et al., 2010). The manipulation of ongoing task absorption would be less likely to affect the retrospective component of prospective memory (remembering to refuel when the flower pot appeared) in the two age groups studied than the prospective component (detecting the flowerpot and then refueling). Following this logic, if retrospective memory processes are driving developmental change in prospective memory (e.g., Smith et al., 2010), then we would not expect ongoing task absorption to play a role in the age effect because it would not likely affect the retrospective component processes. Similarly, cue salience would seem to influence children's ability to detect the cue (prospective component) rather than the retrospective component of prospective memory. The finding of a trend-level interaction between age and cue salience (after controlling for ongoing task performance) is consistent with the idea that cue salience plays a role in age-related increases in prospective memory; however, it is possible that the manipulation of cue salience was too weak and so required fewer executive resources in either condition or simply that the study needed more 
power to detect this interaction effect. Finally, cue centrality had an impact on the prospective component of prospective memory because age and cue centrality interacted. Whether one's attention is directed toward or away from the cue has an obvious impact on cue detection, and having the cue presented in the center of attention boosts younger children's performance to older children's levels. Therefore, the latter two manipulations likely affected the prospective component of prospective memory, whereas ongoing task absorption did not. Given prior evidence that the retrospective memory component might drive some age effects in prospective memory during middle childhood (see also Zöllig et al., 2007, for similar results during adolescence), ongoing task absorption might not have interacted with age because it did not affect the retrospective component of prospective memory, although the current study cannot confirm this directly.

Therefore, a limitation of the current study is its focus on the prospective component of prospective memory compared with the retrospective component. Because all children in the current study were able to report the content of the intention and satisfy our requirement for sufficient retrospective memory (as is standard in prospective memory tasks; see recommendations of Kvavilashvili et al., 2008), this meant that retrospective memory could not be analyzed more carefully. Future work should investigate the extent to which experimental manipulations of executive demand interact with both the prospective and retrospective components of prospective memory.

In terms of future studies, it will be important to follow up on these experimental effects and target the precise nature of the control processes associated with those effects. Although current models do not specify the nature of controlled processes associated with cue centrality in the center of attention, given the differential findings in terms of age interactions across our three experiments, current data argue that the processes associated with overcoming high ongoing task absorption, low cue salience, and cues outside of the center of attention might not rely on the same cognitive resources. Therefore, it might be that ongoing task absorption and cue salience target distinct aspects of controlled processes. For example, perhaps ongoing task absorption and cue salience require lower levels of monitoring, attention, or demands on executive function compared with the cues outside of the center of attention, such that these factors do not tend to interact with age. Instead of concluding that ongoing task absorption and cue salience do not rely on executive processes, we speculate that they require lower levels and might not be the key mechanisms driving age-related improvement in prospective memory. Hence, the processes driving the effects of these two retrieval factors might show a developmental course different from that of prospective memory and, therefore, do not underlie the increase of prospective memory during early school years.

An alternative explanation to the executive account is that the lack of interaction between age and ongoing task absorption, and between age and cue salience, is due to the use of prospective memory cues that are outside of the center of attention. In Experiments 1 and 2, the prospective memory cues corresponded to the outside of the center of attention cues used in Experiment 3. Given that cue centrality interacted with age in Experiment 3, it is possible that the use of outside of the center of attention cues uniquely resulted in prospective memory performance not interacting with age. Future research could examine these issues directly by testing whether measures of executive function predict children's susceptibility to manipulations of ongoing absorption, cue salience, and cue centrality in or outside of the center of attention.

In this vein, the precise cognitive mechanisms that underpin the reported age differences warrant further consideration. As noted previously, there are marked developmental shifts in controlled processing resources in early school-age children, including working memory, inhibitory control, and set shifting (see, e.g., Best et al., 2009, for a review). With regard to parallel prospective memory development, better inhibitory skills in older children possibly promote the ability to stop the ongoing task more effectively for intention execution and to ignore task-irrelevant information. Furthermore, higher working memory capacities in older children are suggested to support monitoring cue information, and shifting skills may support more flexible shifts between execution of the ongoing and prospective tasks. Although the first few published studies examining the developmental role of inhibitory processes in event-based prospective memory have been mixed (e.g., Atance \& Jackson, 2009; Ford et al., 2012; Kerns, 2000; Kvavilashvili et al., 2001; Mackinlay et al., 2009; Mahy \& Moses, 2011), findings for working memory and shifting are rather rare (but see Mahy \& Moses, 2011; Shum et al., 2008). Therefore, future research should address the question of whether age-related differences in the 
integrity of specific executive control operations mediate age-related differences in prospective memory performance more directly. Here, it will be necessary to disentangle the relative importance of key cognitive control facets for prospective memory development.

This was the first attempt to explore mechanisms behind development in prospective memory by contrasting performance in conditions that may rely more or less on controlled processes. Therefore, the purpose of the current study was to lay the groundwork for further examination of the mechanisms involved in prospective memory development during childhood. More work is needed to more thoroughly investigate the role of monitoring in various prospective memory conditions, perhaps by including a condition with no prospective memory cues so that a baseline measure of ongoing task performance can be established. Future studies should continue to examine the role of specific mechanisms (e.g., executive functions) as well as examine interactions among ongoing task absorption, cue salience, and the effect of cue centrality on prospective memory performance. The current study could not examine all of these factors in a fully crossed design, but it is likely that these factors may interact with each other as well as other cognitive abilities. Furthermore, future work could include a baseline block or control condition to examine how resources are devoted to the ongoing task when it is carried out alone compared with in conjunction with the prospective memory task.

To conclude, the current study indicates that there is a developmental increase in prospective memory performance in early school-age children. Furthermore, the degree of difficulty experienced by children in this age group is systematically related to retrieval-based factors. The finding that cue centrality in or outside of the center of attention moderated age differences in prospective performance is consistent with theoretical models that suggest developmental shifts in controlled processing resources as a developmental mechanism in prospective memory functioning.

\section{Acknowledgment}

Preparation of the manuscript was funded partly by a grant from the German Research Foundation (DFG) to M.K. and a Swiss Foreign Student Scholarship to C.E.V.M.

\section{References}

Atance, C. M., \& Jackson, L. K. (2009). The development and coherence of future-oriented behaviors during the preschool years. Journal of Experimental Child Psychology, 102, 379-391.

Best, J. R., Miller, P. H., \& Jones, L. L. (2009). Executive functions after age 5: Changes and correlates. Developmental Review, 29, $180-200$.

Brewer, G. A., \& Marsh, R. L. (2010). On the role of episodic future simulation in encoding of prospective memories. Cognitive Neuroscience, 1, 81-88.

Carlson, S. M. (2005). Developmentally sensitive measures of executive function in preschool children. Developmental Neuropsychology, 28, 595-616.

Cohen, J. (1988). Statistical power analysis for the behavioral sciences. Hillsdale, NJ: Lawrence Erlbaum.

Einstein, G. O., \& McDaniel, M. A. (1990). Normal aging and prospective memory. Journal of Experimental Psychology: Learning, Memory, and Cognition, 16, 717-726.

Einstein, G. O., \& McDaniel, M. A. (2005). Prospective memory: Multiple retrieval processes. Current Directions in Psychological Science, 14, 286-290.

Einstein, G. O., \& McDaniel, M. A. (1996). Retrieval processes in prospective memory: Theoretical approaches and some new findings. In M. Brandimonte, G. O. Einstein, \& M. A. McDaniel (Eds.), Prospective memory: Theory and applications (pp. 115-142). Mahwah, NJ: Lawrence Erlbaum.

Ellis, J. A. (1996). Prospective memory or the realization of delayed intentions: A conceptual framework for research. In M. A. Brandimonte, G. O. Einstein, \& M. A. McDaniel (Eds.), Prospective memory: Theory and applications (pp. 1-22). Mahwah, NJ: Lawrence Erlbaum.

Ellis, J., \& Kvavilashvili, L. (2000). Prospective memory in 2000: Past, present, and future directions. Applied Cognitive Psychology, 14, S1-S9.

Ford, R. M., Driscoll, T., Shum, D., \& Macaulay, C. E. (2012). Executive and theory-of-mind contributions to event-based prospective memory in children: Exploring the self-projection hypothesis. Journal of Experimental Child Psychology, 111, 468-489.

Gathercole, S. E., Pickering, S. J., Ambridge, B., \& Wearing, H. (2004). The structure of working memory from 4 to 15 years of age. Developmental Psychology, 40, 177-190.

Guynn, M. J. (2003). A two-process model of strategic monitoring in event-based prospective memory: Activation/retrieval mode and checking. International Journal of Psychology, 38, 245-256.

Henry, J. D., MacLeod, M. S., Phillips, L. H., \& Crawford, J. R. (2004). A meta-analytic review of prospective memory and aging. Psychology and Aging, 19, 27-39. 
Ihle, A., Hering, A., Mahy, C. E. V., Bisiacchi, P. S., \& Kliegel, M. (in press). Adult age differences, response management, and cue focality in event-based prospective memory: A meta-analysis on the role of task order specificity. Psychology and Aging.

Kerns, K. A. (2000). The CyberCruiser: An investigation of development of prospective memory in children. Journal of the International Neuropsychological Society, 6, 62-70.

Kerns, K. A., \& Price, K. J. (2001). An investigation of prospective memory in children with ADHD. Child Neuropsychology, 7, $162-171$.

Kliegel, M., Altgassen, M., Hering, A., \& Rose, N. (2011). A process-model based approach to prospective memory impairment in Parkinson's disease. Neuropsychologia, 49, 2166-2177.

Kliegel, M., Jäger, T., \& Phillips, L. (2008). Adult age differences in event-based prospective memory: A meta-analysis on the role of focal versus nonfocal cues. Psychology and Aging, 23, 203-208.

Kliegel, M., Mackinlay, R. J., \& Jäger, T. (2008). Prospective memory development: A lifespan approach. In M. Kliegel, M. A. McDaniel, \& G. O. Einstein (Eds.), Prospective memory: Cognitive, neuroscience, developmental, and applied perspectives (pp. 187-216). Mahwah, NJ: Lawrence Erlbaum.

Kliegel, M., McDaniel, M. A., \& Einstein, G. O. (2008). Prospective memory: Cognitive, neuroscience, developmental, and applied perspectives. Mahwah, NJ: Lawrence Erlbaum.

Kvavilashvili, L., \& Ellis, J. (1996). Varieties of intention: Some distinctions and classifications. In M. Brandimonte, G. O. Einstein, \& M. A. McDaniel (Eds.), Prospective memory: Theory and applications (pp. 23-51). Mahwah, NJ: Lawrence Erlbaum.

Kvavilashvili, L., Kyle, F., \& Messer, D. J. (2008). The development of prospective memory in children: Methodological issues, empirical findings, and future directions. In M. Kliegel, M. A. McDaniel, \& G. O. Einstein (Eds.), Prospective memory: Cognitive, neuroscience, developmental, and applied perspectives (pp. 115-140). Mahwah, NJ: Lawrence Erlbaum.

Kvavilashvili, L., Messer, D. J., \& Ebdon, P. (2001). Prospective memory in children: The effects of age and task interruption. Developmental Psychology, 37, 418-430.

Mackinlay, R., Kliegel, M., \& Mäntylä, T. (2009). Predictors of time-based prospective memory in children. Journal of Experimental Child Psychology, 102, 251-264.

Mahy, C. E. V., \& Moses, L. J. (2011). Executive functioning and prospective memory in young children. Cognitive Development, 26, 269-281.

Mäntylä, T., Carelli, M. G., \& Forman, H. (2007). Time monitoring and executive functioning in children and adults. Journal of Experimental Child Psychology, 96, 1-19.

McDaniel, M. A., \& Einstein, G. O. (2000). Strategic and automatic processes in prospective memory retrieval: A multiprocess framework. Applied Cognitive Psychology, 14, 127-144.

McDaniel, M. A., Einstein, G. O., \& Rendell, P. G. (2008). The puzzle of inconsistent age-related declines in prospective memory: A multiprocess explanation. In M. Kliegel, M. A. McDaniel, \& G. O. Einstein (Eds.), Prospective memory: Cognitive, neuroscience, developmental, and applied perspectives (pp. 141-158). Mahwah, NJ: Lawrence Erlbaum.

Meacham, J. A. (1982). A note on remembering to execute planned actions. Journal of Applied Developmental Psychology, 3, $121-133$.

Nigro, G., Senese, V. P., Natullo, O., \& Sergi, I. (2002). Preliminary remarks on type of task and delay in children's prospective memory. Perceptual and Motor Skills, 95, 515-519.

Petermann, F., \& Petermann, U. (2008). Hamburg-Wechsler Intelligenztest für Kinder-IV (HAWIK-IV). Bern, Switzerland: Hans Huber.

Rendell, P. G., Vella, M. J., Kliegel, M., \& Terrett, G. (2009). Effect of delay on children's delay-execute prospective memory performance. Cognitive Development, 24, 156-168.

Schneider, W., \& Bjorklund, D. F. (1998). Memory. In W. Damon, D. Kuhn, \& R. S. Siegler (Eds.). Handbook of child psychology: Cognition, perception, and language (Vol. 2, pp. 467-521). New York: John Wiley.

Schneider, W., \& Pressley, M. (1997). Memory development between 2 and 20 (2nd ed.). Mahwah, NJ: Lawrence Erlbaum.

Scullin, M. K., McDaniel, M. A., \& Einstein, G. O. (2010). Control of cost in prospective memory: Evidence for spontaneous retrieval processes. Journal of Experimental Psychology: Learning, Memory, and Cognition, 36, 190-203.

Scullin, M. K., McDaniel, M. A., Shelton, J. T., \& Lee, J. H. (2010). Focal/nonfocal cue effects in prospective memory: Monitoring difficulty or different retrieval processes? Journal of Experimental Psychology: Learning, Memory, and Cognition, 36, 736-749.

Shum, D., Cross, B., Ford, R., \& Ownsworth, T. (2008). A developmental investigation of prospective memory: Effects of interruption. Child Neuropsychology, 14, 547-561.

Smith, R. E. (2003). The cost of remembering to remember in event-based prospective memory: Investigating the capacity demands of delayed intention performance. Journal of Experimental Psychology: Learning, Memory, and Cognition, 29, 347-361.

Smith, R. E., \& Bayen, U. J. (2004). A multinomial model of event-based prospective memory. Journal of Experimental Psychology: Learning, Memory, and Cognition, 30, 756-777.

Smith, R. E., Bayen, U. J., \& Martin, C. (2010). The cognitive processes underlying event-based prospective memory in school age children and young adults: A formal model-based study. Developmental Psychology, 46, 230-244.

Voigt, V., Aberle, I., Schönfeld, J., \& Kliegel, M. (2011). Time-based prospective memory in school-children: The role of selfinitiation and strategic time-monitoring. Journal of Psychology, 219, 92-99.

Wang, L., Kliegel, M., Liu, W., \& Yang, Z. (2008). Prospective memory performance in preschoolers: Inhibitory control matters. European Journal of Developmental Psychology, 5, 289-302.

Welsh, M., Pennington, B., \& Groisser, D. (1991). A normative developmental study of executive function: A window on prefrontal function in children. Developmental Neuropsychology, 7, 131-149.

Winograd, E. (1988). Some observations on prospective remembering. In M. M. Gruneberg, P. E. Morris, \& R. N. Sykes (Eds.). Practical aspects of memory: Current research and issues (Vol. 1, pp. 348-353). Chichester, UK: Wiley.

Yang, T.-X., Chan, R. C. K., \& Shum, D. (2011). The development of prospective memory in typically developing children. Neuropsychology, 25, 342-352.

Zelazo, P. D., Carlson, S. M., \& Kesek, A. (2008). The development of executive function in childhood. In C. A. Nelson \& M. Luciana (Eds.), Handbook of developmental cognitive neuroscience (pp. 553-574). Cambridge, MA: MIT Press. 
Zelazo, P. D., Müller, U., \& Frye, D. (2003). The development of executive function. Monographs of the Society for Research in Child Development, 68(3, Serial No. 274).

Zöllig, J., West, R., Martin, M., Altgassen, M., Lemke, U., \& Kliegel, M. (2007). Neural correlates of prospective memory across the lifespan. Neuropsychologia, 45, 3299-3314. 